\title{
Premature degenerative shoulder changes in spinal cord injury patients
}

\author{
Santosh Lal
}

Department of Physical Medicine and Rehabilitation, Northwestern University Medical School, Rehabilitation Institute of Chicago, 345 E. Superior Street, Chicago, Illinois 60611, USA

\begin{abstract}
Shoulder pain and the resultant dysfunction is an expected problem in individuals with spinal cord injury. But there is a remarkable lack of information about the natural history, diagnosis, prevention and long term outcomes. Degenerative changes may develop prematurely in their shoulders, due to overuse and altered mechanical stresses, with or without symptoms was the hypothesis of this prospective study. Information from this study is expected to assist in identification of high risk SCI individuals, and ultimately in development of preventive strategies.

The shoulders of 53 spinal cord injury patients from the onset of injury until 15 years duration were subjected to clinical and radiological examination at the Rehabilitation Institute of Chicago. Thirty-eight out of $53(72 \%)$ patients demonstrated radiological evidence of degenerative changes, but only six $(11 \%)$ complained of pain in the shoulders.

This study demonstrated a correlation between individuals with higher level of wheelchair activity $(72 \%)$, higher age $(92 \%$ above and $8 \%$ less than 30 years) and female gender $(89 \%$ females versus $65 \%$ males) more prone to develop degenerative changes in the shoulders. Acromioclavicular joint was predominantly affected.

Detailed radiological findings, etiopathological factors and long term recommendations are discussed. The need for further studies and consistent management strategies are stressed.
\end{abstract}

Keywords: degenerative; etiopathological; mechanical stress; radiological; tetraplegia; spinal cord injury (SCI)

\section{Introduction}

Intensive medical care and comprehensive rehabilitation has enabled the spinal cord injured (SCI) person to live an active, productive life style to almost normal life expectancy. There is a general consensus that upper extremity pain is common in $1 / 3$ to $1 / 2$ of the SCI population which can interfere in their function and independence, ${ }^{1-6}$ yet there is little agreement as to the causes and management techniques. Literature review revealed some 22 retrospective reports with radiological changes in symptomatic patients with upper extremity pain. ${ }^{3,5,6}$ But others reported radiological changes in inactive rather than active individuals, ${ }^{7}$ rejecting the overuse theory proposed by Wing et al. ${ }^{8}$ All published reports indicate the need for further research.

The goal of this prospective study was to systematically assess the shoulders of wheelchair confined SCI person for longer duration, since the onset of injury up to at least for 5 years. The presence or absence of pain was not the criteria for inclusion in the study. All patients who were exposed to putative

Correspondence: S Lal, Oakton Medical Center, 1535 Oakton Street, Des Plaines, Illinois 60018 USA mechanical forces of activities from wheelchair and agreed to participate in the study were included. Theoretically all patients were considered to be at high risk to develop degenerative changes in the shoulders due to altered function of upper extremeties, weight bearing rather than prehensile and placement activities.

\section{Subjects and methods}

Fifty-three subjects, 33 who were tetraplegics and 20 paraplegic, 35 males and 18 females in age range of 19 to 81 years with mean age of 37 years participated in this study. All subjects were initially admitted as inpatients at the Rehabilitation Institute of Chicago (a national center for spinal cord injury) and then were followed in the outpatient department for minimum of 5 and maximum of 15 years.

The subjects were classified into four groups based on the age, gender, level of injury, and the level of wheelchair activities (Table 1). Tetraplegic patients were divided in high (C6 and above) and low (C6 to T1) groups (Table 2). Participants were classified in three groups based on their activities, manual wheelchair independent, manual chair dependant and 
Table 1 Participant subjects

\begin{tabular}{lcccccccccc}
\hline $\begin{array}{l}\text { Subjects } \\
\text { total }\end{array}$ & $\begin{array}{c}\text { Age } \\
19-81\end{array}$ & $M$ & Sex & $F$ & HT & $\begin{array}{c}\text { Injury level } \\
\text { LT }\end{array}$ & PARA & WCD & $\begin{array}{c}\text { Activity level } \\
\text { WCI }\end{array}$ & MWU \\
\hline 53 & Mean & 35 & & 18 & 12 & 21 & 20 & 11 & 39 & 3 \\
& 37 years & & & & & & & & & \\
\hline
\end{tabular}

HT, LT: High and low tetraplegia, PARA: Paraplegia, WCD and WCI: Wheelchair dependent and independent, MWU: Motorized wheelchair user

Table 2 Level of injury versus shoulder changes. Results

\begin{tabular}{|c|c|c|c|c|}
\hline \multirow{2}{*}{$\begin{array}{l}\text { Level of } \\
\text { injury }\end{array}$} & \multicolumn{2}{|c|}{ No. of subjects 53} & \multicolumn{2}{|c|}{ Radiological changes } \\
\hline & No. & & Positive & Negative \\
\hline High tetraplegia & 12 & 22 & $8(15 \%)$ & $4(8 \%)$ \\
\hline Low tetraplegia & 21 & 40 & $15(28 \%)$ & $6(11 \%)$ \\
\hline \multirow[t]{2}{*}{ Paraplegia } & 20 & 38 & $15(28 \%)$ & $5(9 \%)$ \\
\hline & 53 & 100 & $38(72 \%)$ & $15(28 \%)$ \\
\hline
\end{tabular}

Thirty-eight out of $53,(72 \%)$ showed radiological changes in the shoulder joints, 30 out of 38 had low level tetraplegia and paraplegia

motorized chair users (Table 3). Two C6 patients with incomplete tetraplegia who were manual chair independent became partial ambulators with crutches and short leg braces for short distances. One paraplegic patient was using motorized chair due to associated complete brachial plexus injury of nondominant upper extremity and was dependent for transfer activities.

\section{Radiological assessment protocol}

$\mathrm{X}$-rays assessment of both shoulders was done in all 53 participants at the time of initial inpatient admission. Follow up X-rays were done within 6 months of discharge and then every 2 years in the outpatient department. This planned follow up protocol could consistently be accomplished in $60 \%$ of the cases. Additional X-rays were done out of the protocol in individuals with complaints of symptoms.

\section{Results}

These are summarized in Figure 1 and Tables 1,2,3, and 4. Age range of the participants was 19 to 81 with mean age 37 , they were grouped into age higher and younger than 30 years. Individuals in higher age group demonstrated higher incidence of radiographic changes (Figure 1). Thirty-eight of 53 who developed X-ray changes, 35 of them $(92 \%)$ were in higher and only three in the younger $(8 \%)$ age group. Eight of 12 $(15 \%)$ high, 15 of $21(28 \%)$ low tetraplegia and 15 of $20(28 \%)$ of paraplegia group developed degenerative X-ray changes (Table 2). Thirty-four of $39(83 \%)$ manual wheelchair independent, three of $11(27 \%)$ manual chair dependent and one of $3(33 \%)$ motorized chair users demonstrated radiological changes in the Xrays (Table 3 ). Eighteen of 53 subjects were females, 15

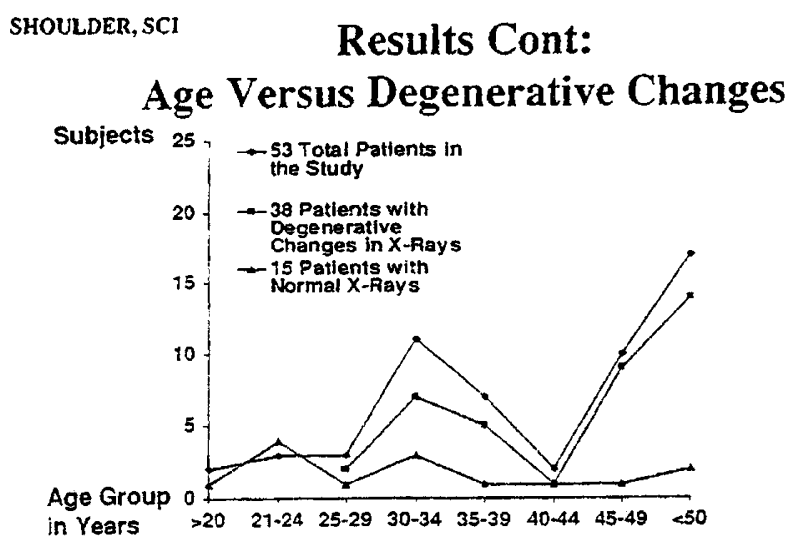

Figure 1 Mean age 37 years. Higher age subjects showed shoulder changes

Table 3 Activity level versus shoulder changes. Results

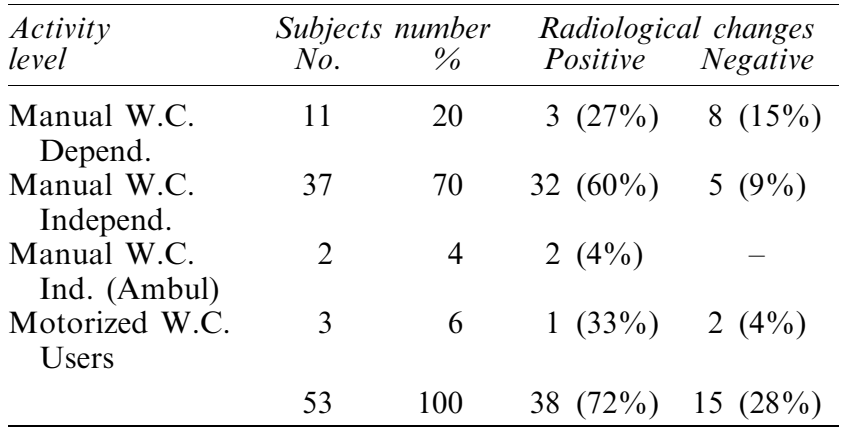

Thirty-nine out of 53 subjects were wheelchair independent and 34 of those had changes in the shoulder joints $(87 \%)$

Table 4 Sex versus degenerative shoulder changes. Results

\begin{tabular}{lcccc}
\hline & \multicolumn{2}{c}{ Subjects } & \multicolumn{2}{c}{ Degenerative changes } \\
Sex & No. & $\%$ & Positive & Negative \\
\hline Male & 18 & 34 & $1528 \%$ & $36 \%$ \\
& & & $(\mathrm{~F} / \mathrm{F} 83 \%)$ & $(\mathrm{F} / \mathrm{F} 17 \%)$ \\
Female & 35 & 66 & $2343 \%$ & $1223 \%$ \\
& & & $(\mathrm{M} / \mathrm{M} 65 \%)$ & $(\mathrm{M} / \mathrm{M} 35 \%)$ \\
& 53 & 100 & $3871 \%$ & $1529 \%$ \\
\hline
\end{tabular}

Fifteen out of 18 female subjects $(83 \%)$ showed radiological joint change. Twenty-three out of 35 male subjects showed positive X-rays $(65 \%)$. Percentages seems different when compared with all 53 patients 
a

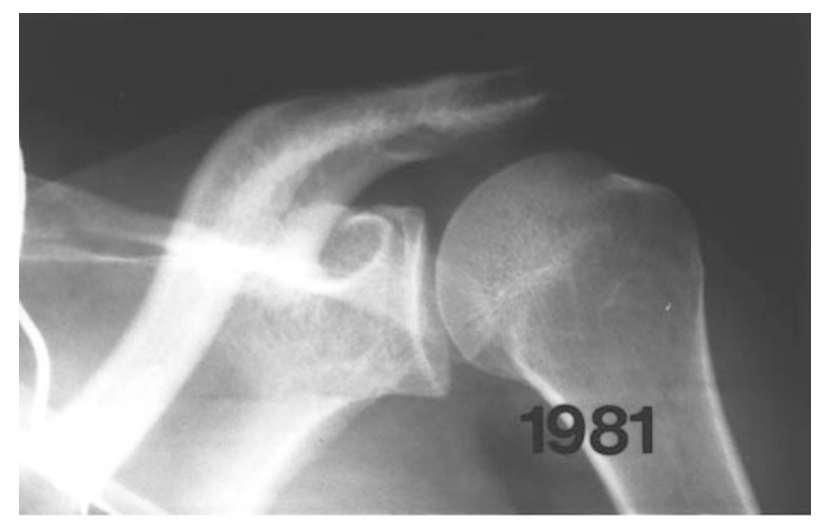

b

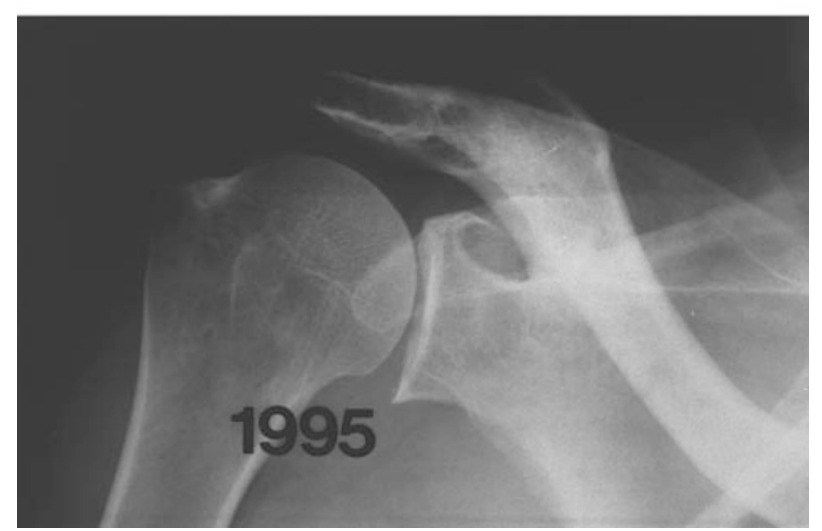

Figure 2 (a)VD 1981 C7 tetraplegia, 1 year post injury. Minimal achromiclavicular spur. (b) VD 1995 narrowing of glenohumeral achromioclavicular joint, spurs, impichment and superior subluxation of humeral head

$(83 \%)$ of them and 23 of 35 males $(66 \%)$ developed Xray changes in their shoulders (Table 4). Interestingly only six of $53(11 \%)$ participants developed shoulder pain. This prospective study was able to document relationships of higher incidence in higher age as proposed by Smith (1989). ${ }^{10}$

Earliest X-ray changes were noted 4 years after the injury in this group. Acromioclavicular joint narrowing (Figure 2a,b) was noted in all 38 subjects (100\%), spur formation $(39 \%)$, necrosis $(34 \%)$, impingement $(29 \%)$ were other significant findings.

\section{Discussion}

Upper extremity pain and deterioration of function is a common problem in a SCI population with a reported prevalence of $1 / 3$ to $1 / 2$, due to over use stress. Although the exact relationship is not clear shoulder dysfunction is thought to increase with age and timesince- injury. Various retrospective studies in the literature have documented conflicting reports. Only symptomatic SCI individuals were included in most of the studies. Nichols et al (1978) ${ }^{1}$, Gellman et al (1988) ${ }^{2}$,

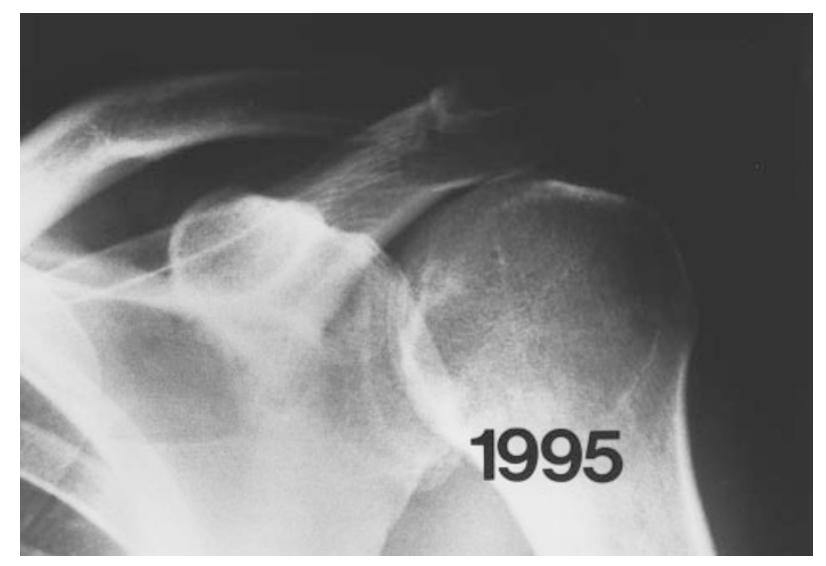

Figure 31995 DT C6 tetraplegia, partial ambulator 10 years post injury. Achromioclavicular changes, achromian spur, impingement. (1991 X-rays were normal)

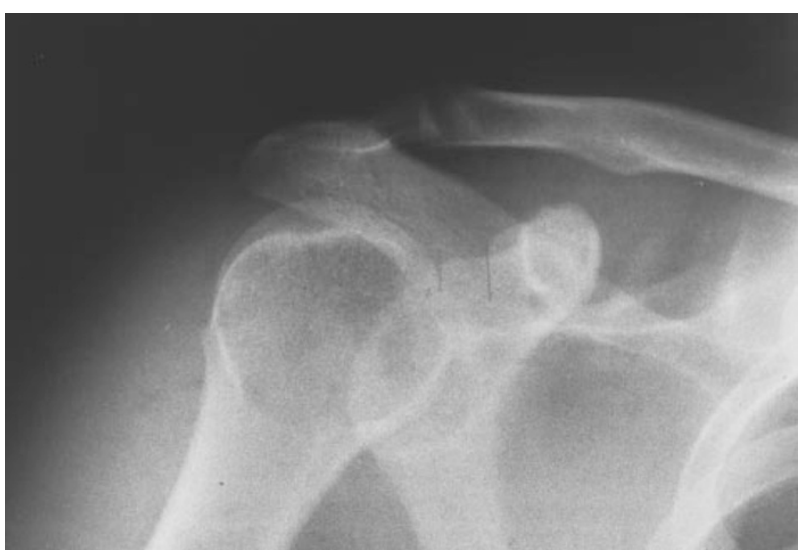

Figure 4 AD C8 tetraplegia. 8 years post injury (Previous X-ray 1989 was normal). Achromiculavicular changes, cystic change humeral head with sclerosis, inferior subluxation of humeral

Subbarao (1994) ${ }^{4}$ and Sie et al (1992) ${ }^{9}$ reported pain in the upper extremities of SCI individuals in $1 / 3$ to $1 / 2$ of their patients based on clinical evaluation. Wing et al $(1983)^{8}$ were not able to prove their overuse theory with no radiographic degenerative shoulder changes in their 10 crutch ambulators of mean age 23.7 years and duration of 8.7 years since injury. Contrary to the overuse theory Wylie et al $(1988)^{7}$ demonstrated radiographic degenerative changes in their 12 of 38 inactive rather than active patients of 20 years duration.

This prospective study was able to document relationship of higher incidence in advanced age (Figure 1). Eight of $53(15 \%)$ less than and 45 of 53 $(85 \%)$ were of higher than 30 years of age. Three of eight $(38 \%)$ and 35 of $45(78 \%)$ subjects with degenerative changes (Figure 1) demonstrates the correlation of higher incidence with higher age. In relation to wheelchair activity 34 of 39 (87\%) 
independents versus (27\%) dependents (Table 2) developed X-ray proven changes in the shoulders. Contrary to the previous reports pain in 30 to $60 \%$ only six of $53(11 \%)$ reported some degree of pain.

Low incidence of pain can only be explained in those with tetraplegia due to impairment of shoulder nerve supply based on Charcot arthropathy Whitneck et al (1993) ${ }^{14}$ as nerve supply to the shoulder joint is from cervical segments (C4-C7) Hollinshead (1988). ${ }^{13}$ Higher incidence of X-ray changes in female gender 15 of $18(83 \%)$ were observed in this study. No such gender prevalence in the literature has been reported though Pentland (1994) $)^{5}$ documented pain in the upper extremities associated with X-ray changes in 11 women was related with paraplegia as age and activity matched non paraplegic females did not develop pain or degenerative changes.

Acromioclavicular joint was found to be most susceptible in X-rays and was involved in all the affected shoulder joints (Figures 2a, b, 3 and 4). Other degenerative changes reported in the literature, spur formation in 39\% (Figure 4), osteonecrosis (Figure 3) $34 \%$ (Barber et al 1996) ${ }^{6}$ and impingement (Figure 4) $29 \%$ (Bayley et al 1987), ${ }^{3}$ were also documented in the shoulder X-rays of subjects of this study. The radiological changes appeared to occur at an early stage than reported in the literature $4-5$ years versus 10 years or longer.

Micro trauma to the cartilage resulting in elaboration of degradative enzymes and causing disturbance in cartilage repair mechanism is the normal process of degenerative joint disease Burr et al (1990), ${ }^{11}$ which is initiated after second decade of life in aging McCarty et al (1993). ${ }^{12}$ This degenerative process might have started in the higher age (30 years and above) group repetitive trauma and badly transmitted forces on the shoulder joint in wheelchair activities might have influenced the rapid progression of degenerative changes resulting into higher incidence of radiographic changes in this group.

\section{Conclusion}

The data from this study suggests that premature shoulder changes appear primarily in wheelchair users of advanced age in less than 10 years with predilection of Acromioclavicular joint. The female individuals may be at a higher risk. Further research is needed to understand the exact etiopathological factors, their clinical relevance and plan for appropriate intervention. Strategy for clinical management was individualized in this study depending on the need of necessary equipment and assistance, to reduce the stress on the shoulders and acceptance by the participants.

\section{Acknowledgements}

Gratitude is extended to Dolores Szymaniak and Dr Roshan Lal of Oakton Medical Center, Desplaines, Illinois, USA, in preparation of this manuscript.

\section{References}

1 Nichols PIR, Norman PA, Ennis J. Wheelchair User's Shoulder Pain in Patients with Spinal Cord Lesions. Scan J Rehabil Med 1978; 11: $29-32$.

2 Gellman H, Sie I, Waters RL. Late Complications of the Weight Bearing Upper Extremity in the Paraplegic Patient. Clin Orthop 1988; 233: $132-135$.

3 Bayley JC, Cochrant TP, Sledge CB. The Impingement Syndrome in Paraplegics. J Bone Joint Surg 1987; 69A: 676-678.

4 Subbarao J, Klopfstein J, Turpin R. Prevalence and Impact of Wrist and Shoulder Pain in Patients with Spinal Cord Injury. $J$ Spinal Cord Med 1994; 18: 9-13.

5 Pentland WE, Twomey LT. Upper Limb Function in Persons with Paraplegia and Implications for Independence. Paraplegia 1994; 32: $211-218$

6 Barbar DB, Gall NG. Osteonecrosis and Overuse Injury of the Shoulder in Paraplegia-Case Report. Paraplegia 1991; 29: 423 426

7 Wylie EJ, Chakera TM. Degenerative Joint Abnormalities in Patients with Paraplegia of Duration Greater than 20 Years. Paraplegia 1988: 26: $101-106$.

8 Wing PC, Tredwell SJ. The Weight Bearing Shoulder. Paraplegia 1983; 21: $107-113$.

9 Sie IH, Waters RL, Adkins RH, Gellman H. Upper Extremity Pain in the Postrehabilitation Spinal Cord Injury Patient. Arch Phys Med Rehabil 1992; 73: $44-48$.

10 Smith I. Aging with Spinal Cord Injury. Rehab Manag 1989; 28 35 .

11 Burr DE, Rodin EL. Trauma as a Factor in the Initiation of Osteoarthritis in Cartilage changes in Osteoarthritis. Brandt KD (ed) Ciba-Giegy 1990; pp 73-80.

12 McCarty DJ, Koopman WJ. Arthritis and Allied Conditions (12th edition), Lea and Febiger Phil, London 1993; pp 1699-1759.

13 Hollinshead W. Anatomy for Surgeons (3rd edition), Harper and Row 1988; 203.

14 Whitneck GG, Charlifue SW, Gerhart KA, Lammertse DP, Manleys, Menter RR, Seedroff KR. Aging with Spinal Cord Injury, New York Demos Publications: 1993 pp 53-72. 